\title{
PREDICTION OF RECURRENT C. DIFFICILE INFECTION
}

The identification of patients who meet three specific criteria may help clinicians pinpoint those who are at risk of recurrent Clostridium difficile infection, according to findings from a new study.

The recurrence of $C$. difficile infection in patients is a major clinical problem that is increasing in incidence. Interestingly, certain individuals are susceptible to repeated infection. This observation led Ciaran Kelly and colleagues to propose that specific host factors might provide an indication of individual risk of recurrent $C$. difficile infection. This proposal led to the development of a clinical prediction rule that identifies patients who have a high risk of recurrent infection.

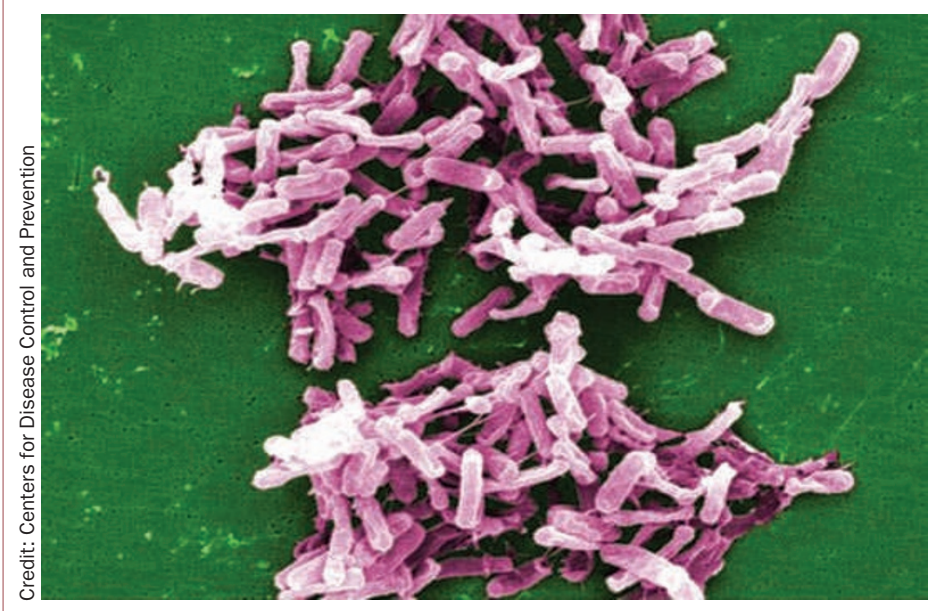

The researchers investigated the clinical characteristics of a cohort of 63 patients to identify factors most strongly associated with the risk of recurrent $C$. difficile infection. The use of multivariate, logistic regression analysis identified three such factors: age $>65$ years, severe or fulminant disease, and continued use of antimicrobial agents after cessation of therapy for $C$. difficile. The ability of these factors to predict recurrence of $C$. difficile infection was then tested prospectively in a second, independent cohort of 89 patients. The researchers found that the presence of these three factors in individual patients predicted whether recurrence of infection would occur with a prognostic accuracy of $72 \%$; “... these three easy-toidentify factors can be used to accurately predict disease outcome," explains Kelly.

The researchers are already using this new prediction tool to identify patients with $C$. difficile infection who are at greatest risk of future recurrence. They anticipate that identification of these patients will alert physicians to the signs and symptoms of recurrence, so recurrent disease can be promptly diagnosed and treated. The researchers hope their model will be used to select individuals at high risk of recurrent $C$. difficile infection for inclusion in future clinical trials to investigate the ability of new agents, such as probiotics, novel antimicrobial agents, vaccination or immunotherapy, to prevent such infection.

\section{Susan J. Allison}

Original article Hu, M. Y. et al. Prospective derivation and validation of a clinical prediction rule for recurrent Clostridium difficile infection. Gastroenterology 136, 1206-1214 (2009). 